\title{
The Measurement of School Sports Atmosphere and the Relation Between Middle School Students Scientific Creativity
}

\author{
Peng Hou*, Shaoyong Yu \\ Department of Physical Education, Xidian University, Xi'an, China, 710071 \\ Email: yushaoyong@126.com
}

\begin{abstract}
Purpose - This paper builds the structure dimension of the school sports atmosphere, develops the corresponding measuring tool, discusses the relation between the school sports atmosphere and the scientific creativity of middle school students. Methods - Research methods combining theory with empirical evidence, including interview survey method, psychological measurement method, mathematical statistics, etc. Conclusion — The school sports atmosphere covers 13 items in three dimensions: self-identification, teacher-student trust, and school environment. The overall Cronbach $\alpha$ value of the scale is 0.865 . The two dimensions of self-identification and teacher-student trust can significantly positively predict the product improvement ability, experimental design ability and technical product design ability of middle school students' scientific creativity. School environment can positively predict the seven dimensions of scientific creativity of middle school students.
\end{abstract}

Keywords: school sports atmosphere, middle school students, scientific creativity

Atmosphere is a series of people's perception of working and living environment, which is measurable and affects people's behavior and motivation (Litwin \& Stringer, 1968) ${ }^{[1]}$. Peicun Cai (1985) ${ }^{[2]}$ pointed out that the atmosphere comes from the overall perception of the environment by internal members. Weizheng Chen $(2005){ }^{[3]}$ believes that the atmosphere can measure the unique persistence style of the environment within a certain range. Yuandong Gu and Jisheng Peng (2010)

${ }^{[4]}$ mentioned that the atmosphere is a long-term trait formed in the overall environment, which can be directly or indirectly perceived by internal members, and can be recognized through the measurement of trait attribute elements. This study uses the relevant definition of atmosphere in the field of organization for reference, and adopts the method of adding varieties and differences to define school sports atmosphere as an atmosphere or environment with certain characteristics gradually formed in the school, which can be perceived and recognized by teachers and students and can affect their exercise behavior and motivation. Different from general abilities, scientific creativity emphasizes its novelty and originality. Weiping $\mathrm{Hu}(2002)^{[5]}$ pointed out that the core of scientific creativity is scientific creative thinking, and scientific creativity is influenced by external factors such as culture, society, environment, family and school. Jinzhen Li (2004) ${ }^{[6]}$, Anna (2015) ${ }^{[7]}$, Nicholas (2016) ${ }^{[8]}$, Wang (2016) ${ }^{[9]}$ et al. found that school and family environment has direct and indirect influences on creativity. Scientific creativity develops with the development of individual characteristics, and school environment plays an important and unique role in the development of children and adolescents' creativity. This study mainly uses the definition of Professor Weiping Hu, which is considered to be the unity of the three-dimensional combination of creative process, product and quality. How does building a strong school sports atmosphere affect the educational development of scientific creativity of middle school students? It is of great significance to promote and strengthen the importance of school sports in the context of the continuous high pressure of the high school enrollment rate in China. It is of great significance to promote the overall healthy growth of young people and to achieve innovative countries and healthy China. In addition, it is helpful to deeply understand the dynamic interaction and relationship between middle school students' creativity development and school environment, and enrich and develop the theories of adolescent developmental psychology.

\section{Survey object}

Students from the high school affiliated to northwest polytechnic university, Xi'an Aichi Middle School and Xi'an Gaoxin No.1 Middle School were studied as samples. These three middle schools have excellent academic atmosphere, good sports environment and facilities, and pay attention to the combination of school sports work and physical education. The sampling is carried out in the principle of integrated sampling and convenient sampling. Since the first grade of junior middle school is a critical period for the development of creative tendency (Chongde Lin, 2012) ${ }^{[10]}$, this study mainly selects a total of 400 middle school students (male 211, female 189) in the second grade of junior middle school as 
samples, with the average age of students being 14.3 years old.

\section{Research ideas and methods}

\subsection{Research ideas}

Based on the preliminary literature to construct the structural dimension of school sports atmosphere and the theoretical connection with creativity and put forward relevant assumptions, develop the school sports atmosphere measurement tools, and use the empirical research method to test the hypothesis.

\subsection{Interview survey method}

According to the needs of research and theoretical framework, five school administrators, eight teachers, thirteen students and seven students' guardians (parents or relatives) were interviewed in an open non-structured way according to the concept, content or presentation of school sports atmosphere. The interview ends when no new information emerges from the interviewees' answers to the questions.

\subsection{Psychometric method}

\subsubsection{Measurement of school sports atmosphere}

The information obtained from the interviews will be sorted out and analyzed. At the same time, combined with the relevant research of the predecessors, the dimensions of the school sports atmosphere will be theoretically constructed, and the scale of the school sports atmosphere will be preliminarily designed, and experts will be invited to make corrections. At the same time, five psychologists and five sports experts were selected to evaluate the questionnaire items and make recommendations. After further revision, they were used for formal measurement.

\subsubsection{Measurement of scientific creativity}

There are 7 questions in the Adolescent Science Creativity Test, each question is followed by a Q\&A demonstration. Pearson product moment correlation coefficient is between 0.793 and 0.913 , and the reliability of the whole scale is 0.857 . Meanwhile, the test has high structural validity (Jiliang Shen, 2002) ${ }^{[11]}$.

A total of 400 questionnaires were distributed, and invalid questionnaires were excluded. 397 valid questionnaires were processed using SPSS18.0 and AMOS20.0. The main methods were project analysis, exploratory factor analysis, confirmatory factor analysis, cross-sample test and multiple linear regression analysis.

\section{Results and analysis}

\subsection{Construction of the school sports atmosphere scale}

\subsubsection{Theoretical basis}

Early mature organizational climate scale includes the KEYS scale developed by Harvard University's Amabile (1996) ${ }^{[12]}$. It is considered that atmosphere is a description of internal personnel's perception of their environment, and self-report scale can be used to measure internal personnel's perception of relevant atmosphere, including organizational creativity, freedom, pressure and organizational obstacles to creativity. Bock (2005) ${ }^{[13]}$ et al. measured and tested organizational atmosphere from three aspects: friendly relationship, innovative atmosphere and fair atmosphere. Yun Liu (2010) ${ }^{[14]}$ measured the organizational atmosphere from the dimensions of colleague support, supervisor support and organizational support, and developed a measurement scale for 15 items. After a series of tests, it has good reliability and validity. Xianya Wang $(2014)^{[15]}$ measured the organizational atmosphere from the four dimensions of trust, communication, emotion and fairness in the study of organizational atmosphere. The results show that the scale of 12 items has good reliability and validity. This study mainly refers to the dimensions involved in the above research and combines the information obtained from the interview to design the entry pool of the initial measurement scale of school physical education atmosphere.

\subsubsection{Exploratory factor analysis}

Exploratory factor analysis was conducted using the SPSS18.0 software for the test results of 13 items in the initial measurement table of the school sports atmosphere. The subjects were 200 middle school students. Before factor analysis, the conformity of $\mathrm{KMO}$ value was tested, and $\mathrm{KMO}=0.837(>0.5)$ was obtained, suitable for EFA analysis (Minglong $\mathrm{Wu} 2003)^{[16]}$. In addition, the data from Bartlett's spherical test $\chi 2=466.402, \mathrm{df}=78, \mathrm{p}<0.001$ indicates that there is a common factor between the population correlation matrices, which is suitable for factor analysis. By means of principal component analysis and maximum variance method of orthogonal rotation, factor extraction was carried out for the measurement of school physical education atmosphere scale. Three common factors with eigenvalues greater than 1 in 
explain total variance scale, and $57.948 \%$ of explain total variance.

Based on previous studies and the definition of school sports atmosphere in this paper, three common factors formed by exploratory factor analysis are named as: self-cognition, teacher-student trust and school environment. Selfidentification is a kind of consciousness that exists in people's minds. It is an understanding of the atmosphere or value of sports. It can guide the brain to make corresponding judgments and choices of the surrounding overall atmosphere. This dimension is mainly considered from the following aspects. We will be happy to exercise with others; exercise is a responsibility to yourself; physical exercise is easy to integrate into the group; physical exercise relieves stress; it will be very happy to help people do physical exercise together. Teacher-student trust is a positive expectation and judgment between teachers and students in physical education or extracurricular exercise. It is the basic premise for mutual communication and cooperation. Based on trust, teachers can guide students to complete physical education content, narrow the distance between teachers and students, enable students to give full play to their sports potential, and thus form a good atmosphere for classroom and extracurricular exercise atmosphere. This dimension is mainly considered through the following aspects. : The teacher encourages everyone to do physical exercise; the teacher guides us to discover the benefits of physical exercise and more exercise methods; the teacher and everyone exercise together, the atmosphere is very active. The school environment is a general term for school sports hardware facilities and school sports soft power in a macro sense. It also includes the school's emphasis on sports work and the implementation of policies. This dimension is mainly considered through the following aspects: the traditional culture of the school infects everyone to exercise spontaneously; the school's exercise facilities make everyone interested in exercise; the diversity of the curriculum allows us to choose the projects we are interested in. Many faculty members also get involved.

Table 1 shows the basic situation of the subscales and total tables of the school sports atmosphere that were initially determined after exploratory factor analysis.

Table 1. Cronbach alpha of each sub-scale of school sports atmosphere

\begin{tabular}{cccc}
\hline Scale dimension & Number of participants & Number of entries & Cronbach $\alpha$ \\
Self-identification & 200 & 5 & 0.784 \\
Teacher-student trust & 200 & 4 & 0.774 \\
School environment & 200 & 4 & 0.744 \\
Total tables & 200 & 13 & 0.889 \\
\hline
\end{tabular}

\subsubsection{Project Analysis}

Table 1 shows the sample size, mean, standard deviation, correlation of other questions, total correlation of questions, total correlation of correction questions, and $\mathrm{CR}$ value (P value) of each item score in the initial test. After the relevant analysis, the values of each indicator show good and can be retained. According to Minglong Wu (2003) ${ }^{[16]}$, in this study, the standard deviation of each measurement item score is greater than 0.8 , so according to the actual research, all the items with standard deviation greater than 0.8 are retained.

\subsubsection{Structural model test of school sports atmosphere scale}

The items retained after project analysis and EFA analysis were used for the structural model analysis. The AMOS 20.0 software was used, and the sample number was 200 . The maximum likelihood method was used, and the output showed that all the coefficients reached a significant level. According to the View Text For Model, the $P$ value reached a very significant level $(P<0.01)$, and the $\chi^{2} / d f$ value was 1.731 , conforming to the goodness-of-fit criteria of less than 5 and 2 or 3. In other goodness-of-fit indicators, the RMR is $0.031(<0.05)$, and the fitting degree is good. The AGFI, NFI, IFI, and CFI indicators are all above 0.90 , more than 0.90 , and the GFI is close to 0.90 . This test is considered to meet the standard of good fit with the existence of allowable error. In addition, the value of RMSEA is 0.067 , which is an acceptable fit. In general, the overall goodness of fit of the model is good.

\subsubsection{Cross-sample test of the school sports atmosphere scale}

Using the data of the remaining 197 samples for cross-sample testing, the Cronbach $\alpha$ values of the three dimensions of self-identification, teacher-student trust, and school environment were all greater than 0.7 , and the overall Cronbach $\alpha$ coefficient was 0.865 . It can be seen that both the subscale and the total scale achieve a good reliability coefficient. As can be seen from the above data, the school sports atmosphere scale has reached the expected reliability standard.

The structural model analysis was continued for 13 items from 197 scales tested. The statistical software is AMOS20.0, and the method is Maximum likelihood method (ML).The input data type is the original data. The output is 
shown in Figure 1.

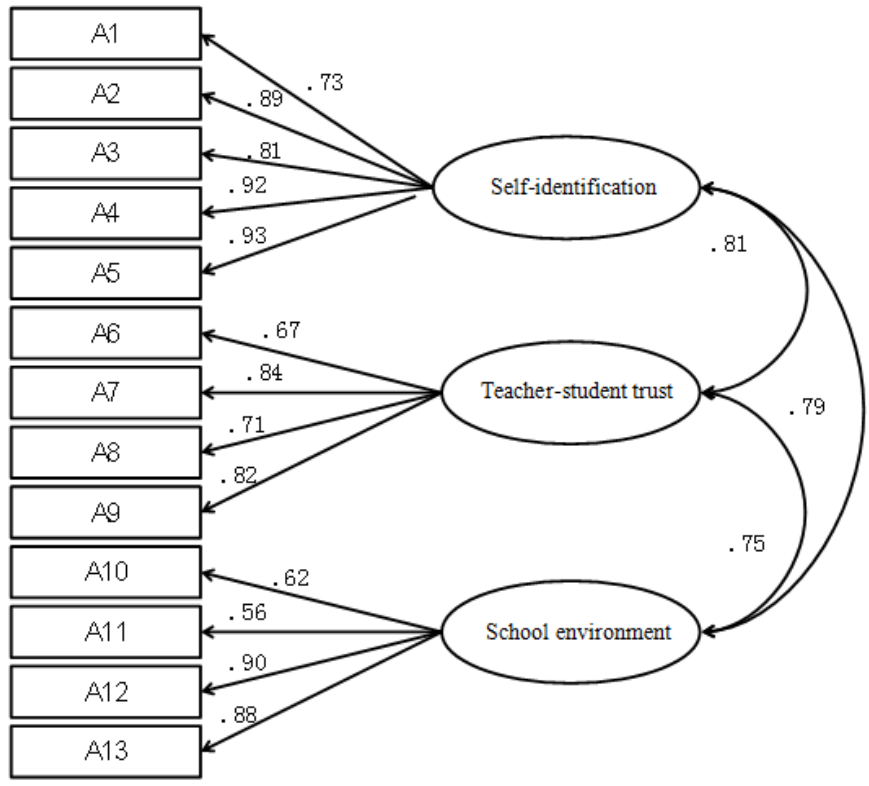

Figure 1. Second order three factors of a cross-sample verification model in school sports atmosphere

The AMOS20.O cross-sample validity verification results of the school sports atmosphere scale showed that the $P$ value reached a significant level $(<0.05)$, and the $\chi^{2} / \mathrm{df}$ value was 1.596 , which was in line with the goodness-of-fit criteria of less than 5 and 2 or 3 . On other goodness-of-fit indicators, the RMR was $0.046(<0.05)$, and the NFI, IFI, and CFI indicators were all above 0.90. The GFI and AGFI indices were acceptable. The RMSEA value is 0.083 . As can be seen from Figure 1, the normalized loads of the three dimensions (self-identification, teacher-student trust, school environment) in this sample are both high, between 0.56 and 0.93 . It is concluded that the observed variables have a good interpretation rate with the corresponding latent variables. Overall, the overall goodness of fit of the model is acceptable.

\subsection{The influence of school sports atmosphere on the scientific creativity of middle school students}

\subsubsection{Proposal of relevant hypotheses}

William (1966) ${ }^{[17]}$ argues that the creativity of middle school students is closely related to the school atmosphere. The research of Chang $(2013)^{[18]}$ indicates that the environment and atmosphere of learning can affect an individual's creativity. Relevant research involving the relationship between school sports and creativity has the following results: the creativity of college students has a significant positive correlation with play games (Tseng, 2002) ${ }^{[19]}$, play and games contribute to the formation of creativity, the class atmosphere related to playing games is positively correlated with students' drawing and language creativity, and "cooperation and friendship" in the class playing game atmosphere can directly predict students' creativity (Chang, 2013). Yanfang Li (2009) ${ }^{[20]}$ pointed out that the campus activities of art, sports and knowledge can not only promote the development of divergent thinking, but also contribute to the deepening of creative consciousness. Based on the above theoretical basis, this study proposes the hypothesis that there is a positive predictive effect between school physical education atmosphere and every dimension of scientific creativity. The testing framework is shown in Figure 2. The School Sports Climate Scale developed by the study and the Middle School Science Creativity Test Scale developed by Professor Jiliang Shen were used to measure and collect data. 


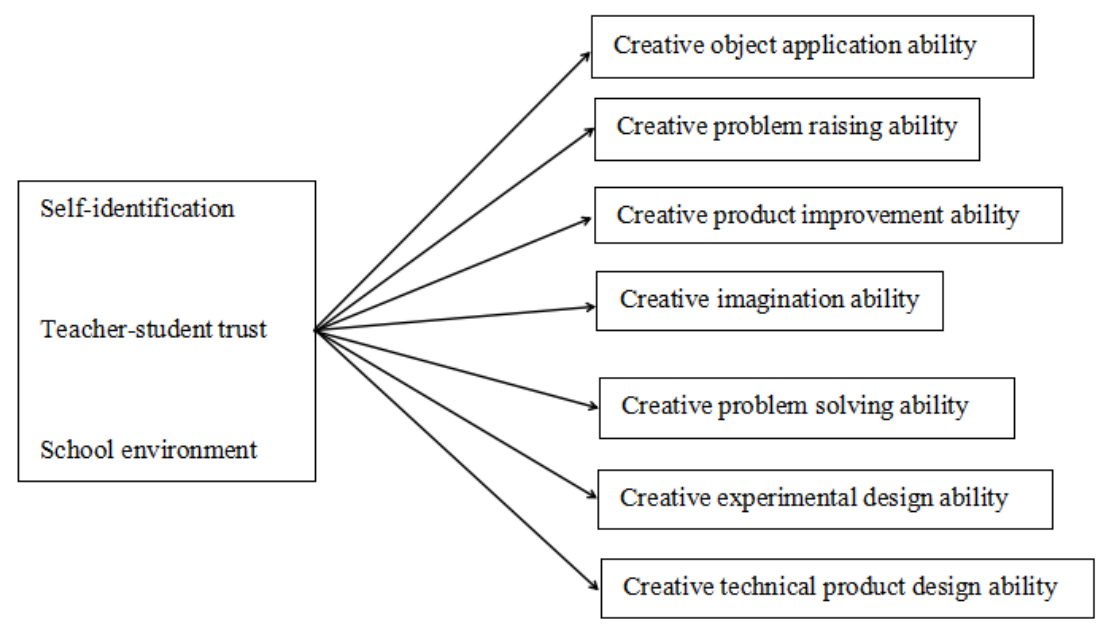

Figure 2. School sports atmosphere and scientific creativity concrete inspection frame

The three dimensions of self-identification, teacher-student trust and school environment are the independent variables, and the seven dimensions of the scientific creation power table are used as the dependent variables to develop multiple linear regression analysis to verify the correlation between independent variables and dependent variables.

3.2.2 Multiple linear regression analysis of school sports atmosphere and creative object application ability

After multiple linear regression, the explanatory power of the three dimensions (self-identification, teacher-student trust, and school environment) in the school sports atmosphere for the application ability of creative objects is $62.2 \%$ $\left(R^{2}=0.622 ; \mathrm{F}=52.672 ; P<0.01\right)$. The results are shown in Table 2 .

Table 2. The multivariate regression analysis of the creative object application to school sports atmosphere

\begin{tabular}{cccccccc}
\hline Independent variable & Estimated value of B & Standard error & Beta & $t$ & $p$ & Tolerance & VIF \\
\hline Self-identification & -0.309 & 0.228 & -0.110 & -1.357 & 0.178 & 0.603 \\
Teacher-student trust & 0.103 & 0.190 & 0.045 & 0.540 & 0.591 & 0.560 & 1.7859 \\
School environment & 1.406 & 0.118 & 0.790 & 11.905 & 0.000 & 0.894 & 1.119 \\
\hline
\end{tabular}

As shown in Table 2, the estimated value of self-identification dimension B is -0.309 , with a significance of 0.178 . The estimated value of teacher-student trust dimension B is 0.103 , with a significance of 0.591 . The estimated value of school environment dimension B is 1.406 , with significance of 0.000 . Both tolerance and VIF meet the requirement that there is no collinearity problem. It shows that self-identification and teacher-student trust have no significant positive predictive effect on creative object application ability. It does not support the hypothesis proposed in the paper. The school environment has a significant positive predictive effect on creative object application ability, supporting the hypothesis proposed in the paper.

3.2.3 Multiple linear regression analysis of school sports atmosphere and creative problem raising ability

After multiple linear regression, the three dimensions of the school sports atmosphere (self-identification, teacherstudent trust, school environment) have a capacity explanatory power of $47.9 \%$ for creative problems $\left(R^{2}=0.479 ; \mathrm{F}=29.380\right.$; $P<0.01)$. The results are shown in Table 3 .

Table 3. The multivariate regression analysis of the creative problem-making ability to school sports atmosphere

\begin{tabular}{cccccccr}
\hline Independent variable & Estimated value of B & Standard error & Beta & $t$ & $p$ & Tolerance & VIF \\
\hline Self-identification & -0.347 & 0.187 & -0.176 & -1.856 & 0.067 & 0.603 \\
Teacher-student trust & 0.272 & 0.156 & 0.172 & 1.742 & 0.085 & 0.560 \\
School environment & 0.813 & 0.097 & 0.654 & 8.387 & 0.000 & 0.894 & 1.787
\end{tabular}

As shown in Table 3, the estimated value of self-identification dimension B is -0.347 , and its significance is 0.067 . The estimated value of teacher-student trust dimension B is 0.272 , and its significance is 0.085 . The estimated value of school 
environment dimension B is 0.813 , and the significance is 0.000 . Tolerance and VIF values show no collinearity problems. It indicates that self-identification and teacher-student trust have no significant positive prediction effect on creative problem raising ability, which not support the hypothesis put forward in the paper. School environment has a significant positive prediction effect on creative problem raising ability, which support the hypothesis put forward in the paper.

\subsubsection{Multiple linear regression analysis of school sports atmosphere and creative product improvement ability}

After multiple linear regression, the explanatory power of the three dimensions (self-identification, teacher-student trust, and school environment) in school physical education atmosphere for creative product improvement ability is $61.6 \%$ $\left(R^{2}=0.616 ; \mathrm{F}=51.391 ; P<0.01\right)$. The tolerance and VIF values meet the requirement of no collinearity problem. The results are shown in Table 4.

Table 4. The multivariate regression analysis of the creative product improvement ability to school sports atmosphere

\begin{tabular}{cccccccc}
\hline Independent variable & Estimated value of B & Standard error & Beta & $t$ & $p$ & Tolerance & VIF \\
\hline Self-identification & -0.423 & 0.190 & -0.181 & -2.226 & 0.028 & 0.603 \\
Teacher-student trust & 0.357 & 0.158 & 0.190 & 2.254 & 0.026 & 0.560 & 1.787 \\
School environment & 1.092 & 0.098 & 0.742 & 11.092 & 0.000 & 0.894 & 1.119 \\
\hline
\end{tabular}

As shown in Table 4, the estimated value of self-identification dimension B is -0.423 , and its significance is 0.028 . The estimated value of teacher-student trust dimension B is 0.357 , and its significance is 0.026 . The estimated value of school environment dimension B is 1.092, and its significance is 0.000 . It shows that self-identification, teacher-student trust and school environment have a significant positive predictive effect on creative product improvement ability, supporting the hypothesis proposed in the paper.

\subsubsection{Multiple linear regression analysis of school sports atmosphere and creative imagination ability}

After multiple linear regression, the explanatory power of the three dimensions (self-identification, teacher-student trust, and school environment) in school sports atmosphere for creative imagination ability is $48.1 \%\left(R^{2}=0.481 ; \mathrm{F}=29.703\right.$; $P<0.01$ ), and there is no col-linearity problem. The test results are shown in Table 5.

Table 5. The multivariate regression analysis of the creative imagination to school sports atmosphere

\begin{tabular}{cccccccc}
\hline Independent variable & Estimated value of B & Standard error & Beta & $t$ & $p$ & Tolerance & VIF \\
\hline Self-identification & -0.160 & 0.149 & -0.101 & -1.070 & 0.287 & 0.603 \\
Teacher-student trust & 0.072 & 0.124 & 0.057 & 0.576 & 0.566 & 0.560 & 1.787 \\
School environment & 0.686 & 0.077 & 0.690 & 8.879 & 0.000 & 0.894 \\
\hline
\end{tabular}

Table 5 shows that the estimated value of self-identification dimension B is -0.160 , with a significance of 0.287 . The estimated value of teacher-student trust dimension B is 0.072 , with a significance of 0.566 . The estimated value of school environment dimension B is 0.686 , with the significance is 0.000 . It is believed that self-identification and teacher-student trust have no significant positive prediction effect on creative imagination ability, which does not support the hypothesis put forward in this paper. School environment has a significant positive prediction effect on creative imagination ability, which supports the hypothesis put forward in this paper.

3.2.6 Multiple linear regression analysis of school sports atmosphere and creative problem solving ability

After multiple linear regression, the three dimensions of the school sports atmosphere (self-identification, teacherstudent trust, school environment) have an explanatory power of $70.9 \%$ for creative problem-solving ability $\left(\mathrm{R}^{2}=0.709\right.$; $\mathrm{F}=77.955 ; \mathrm{P}<0.01)$. And there is no col-linearity problem. The test results are shown in Table 6 .

Table 6. The multivariate regression analysis of the creative problem-solving ability to school sports atmosphere

\begin{tabular}{cccccccc}
\hline Independent variable & Estimated value of B & Standard error & Beta & $\mathrm{t}$ & $\mathrm{p}$ & Tolerance & VIF \\
\hline Self-identification & -0.227 & 0.144 & -0.112 & -1.578 & 0.118 & 0.603 \\
Teacher-student trust & 0.208 & 0.120 & 0.128 & 1.733 & 0.086 & 0.560 \\
School environment & 1.042 & 0.074 & 0.816 & 14.007 & 0.000 & 0.894 & 1.119 \\
\hline
\end{tabular}


Table 6 shows that the estimated value of self-identification dimension B is -0.227 , and its significance is 0.118 . The estimated value of teacher-student trust dimension B is 0.208 , and its significance is 0.086 . The estimated value of school environment dimension B is 1.042 , and its significance is 0.000 . It is believed that self-identification and teacherstudent trust do not play a significant positive predictive role in creative problem-solving ability, which does not support the hypothesis proposed in this paper. School environment plays a significant positive predictive role in creative problemsolving ability, which supports the hypothesis proposed in this paper.

\subsubsection{Multiple linear regression analysis of school sports atmosphere and creative experimental design ability}

After multiple linear regression, the explanatory power of creative experiment design ability in the three dimensions of school sports atmosphere (self-identification, teacher-student trust, school environment) was $24.8 \%\left(R^{2}=0.248\right.$; $\mathrm{F}=10.536 ; P<0.01)$. The tolerance and VIF values show no col-linearity problems. The test results are shown in Table 7 .

Table 7. The multivariate regression analysis of the creative experimental design ability to school sports atmosphere

\begin{tabular}{cccccccc}
\hline Independent variable & Estimated value of B & Standard error & Beta & $t$ & $p$ & Tolerance & VIF \\
\hline Self-identification & -0.982 & 0.480 & -0.233 & -2.044 & 0.044 & 0.603 \\
Teacher-student trust & 0.940 & 0.401 & 0.278 & 2.346 & 0.021 & 0.560 & 1.659 \\
School environment & 1.067 & 0.249 & 0.401 & 4.287 & 0.000 & 0.894 & 1.119
\end{tabular}

Table 7 shows that the estimated value of self-identification dimension B is -0.982 , with a significance of 0.044 . The estimated value of teacher-student trust dimension B is 0.940 , with significance of 0.021 . The estimated value of school environment dimension B is 1.067 , with a significance of 0.000 . The test data shows that self-identification, teacher-student trust and school environment have significant positive predictive effects on creative experimental design ability, supporting the relevant hypotheses proposed in this paper.

3.2.8 Multiple linear regression analysis of school sports atmosphere and creative technical product design ability

After multiple linear regression, the explanatory power of the three dimensions (self-identification, teacher-student trust, and school environment) in school sports atmosphere for creative technical product design ability is $88.1 \%\left(R^{2}=0.881\right.$; $\mathrm{F}=237.247 ; P<0.01)$. Tolerance and VIF values show no collinearity problems. The test results are shown in Table 8 .

Table 8. The multivariate regression analysis of the creative technical product design ability to school sports atmosphere

\begin{tabular}{ccccccccc}
\hline Independent variable & Estimated value of B & Standard error & Beta & $t$ & $p$ & Tolerance & VIF & \\
\hline Self-identification & -0.738 & 0.355 & -0.940 & -2.075 & 0.041 & 0.603 \\
Teacher-student trust & 0.696 & 0.297 & 0.110 & 2.345 & 0.021 & 0.560 & 1.787 \\
School environment & 4.537 & 0.184 & 0.917 & 24.633 & 0.000 & 0.894 & 1.119 \\
\hline
\end{tabular}

As shown in Table 8, the estimated value of self-identification dimension B is 0.738 , and its significance is 0.041 . The estimated value of teacher-student trust dimension B is 0.696 , and its significance is 0.021 . The estimated value of school environment dimension B is 4.537 , and its significance is 0.000 . It can be seen from the data that self-identification, teacher-student trust and school environment have significant positive predictive effects on creative technical product design ability, supporting the relevant hypotheses proposed in this paper.

\subsection{Discussion}

After a series of tests, the resulting school sports atmosphere scale covers three dimensions: self-identification, teacher-student trust, and school environment. It is not only related to the dimensions of atmosphere scale constructed by Bock (2005) ${ }^{[13]}$, Yun Liu (2010) ${ }^{[14]}$ and Xianya Wang (2014) ${ }^{[15]}$, but also reflects some unique characteristics of school sports. The five items of self-cognition mainly reflect the students' cognition process of school sports atmosphere, including the feeling, perception, memory, thinking and other psychological phenomena of school sports atmosphere. The four items of teacher-student trust mainly reflect the expected judgment of ability, goodwill and notarial consistency between middle school teachers and students. The four items of the school environment include the sum of various natural and material conditions on which the majority of teachers and students of the school rely on physical education activities, sports training, and sports competitions. It not only meets the needs of teachers and students for sports activities and fitness 
in physical form, but also influences the emotions and hearts of teachers and students all the time to a large extent. It is an important part of the construction of excellent campus cultural atmosphere. It can be seen that school sports atmosphere is the integration of teachers and students' perception of sports spiritual level and campus material environment level.

In the relationship between school sports atmosphere and student creativity, the results of multiple linear regression analysis show that the two dimensions of teacher-student trust and school environment are closely related to the creativity of middle school students. Zhe Gong (2017) ${ }^{[21]}$ also points out that trust and creativity are both important research objects in psychology, and trust has three effects on creativity: stimulation (positive promotion), inhibition (negative inhibition) and inverted $U$ (dessert effect). At the same time, he also points out that more attention should be paid to the effect of the mechanism of trust and other related variables on creativity. According to Yunfeng Liu (2010) ${ }^{[22]}$, graduate students' trust in their tutors has a significant positive impact on graduate students' creativity, and graduate students' trust in their tutors has a partially positive regulating effect on the relationship between supervisor support behavior and graduate students' creativity. Thus it can be seen that teacher-student trust has an important impact on the creativity of middle school students.

According to the results of this study, school sports environment is also closely related to the creativity of middle school students. In recent years, some scholars have put forward the view that "environment and atmosphere itself are an essential part of creativity". Fleith $(2000){ }^{[23]}$ studied the learning factors that influence the creativity of science. The results show that the psychological security environment helps students to exert their creativity. Donaldson (2016) ${ }^{\text {[24] }}$ takes sports as a part of scientific principles to explore diverse creative learning, believing that an open sports classroom atmosphere is conducive to cultivating students' curiosity and adventure spirit, and students' divergent thinking ability develops more strongly. In addition, this phenomenon also conforms to the ecosystem theory of adolescent development. Brown-fenbrunner's ecosystem theory makes a detailed analysis of the impact of the environment, and believes that biological factors and environmental factors interact to affect human development. Scholar Bandura also believes that the environment not only affects the development of individuals, but also is affected by the development of individuals. In addition, this dimension is more inclined to the category of sports practice, which further explains the role of exercise behavior in the development of middle school students' creativity. Both Lubant's "multi-factor theory of creativity" and Amabile's theory believe that creativity is a product of environmental effects. Environmental factors of society, family and school have important influences on the performance of creativity, enabling more people to actively exert their creative talents. Based on this, it can be determined that the campus sports environment, whether it is the school sports building facilities, or the sports classroom learning environment, or the atmosphere in which the surrounding people participate in sports, will have an impact on the cognition of middle school students, and the impact on students' personality and problem-solving ability and other aspects should be subtle. It further reminds us that under the general goal of promoting moral education, the whole society should be more aware of the necessity and urgency of doing well in school physical education, and further enhance students' innovative spirit by building a positive school physical education atmosphere, shaping students' active mind, healthy body and happy mood.

\section{Conclusions and recommendations}

The school sports atmosphere scale includes 3 dimensions (self-identification, teacher-student trust and school environment, with a total of 13 items. The Cronbach $\alpha$ value is 0.865 . Among them, self-identification dimension included 5 items, with Cronbach $\alpha$ value of 0.782. Teacher-student trust dimension includes 4 items, with Cronbach $\alpha$ of 0.737 . School Environment dimension includes 4 items, with Cronbach $\alpha$ value of 0.715 . The scale has good reliability and validity and can be used as a measurement tool in future related research.

The school environment has a significant positive predictive effect on the seven dimensions of scientific creativity of middle school students (object application ability; problem presentation ability; product improvement ability; imagination ability; problem solving ability; experimental design ability; technical product design ability). Self-identification and teacher-student trust have a significant positive predictive effect on the product improvement ability, experimental design ability and technical product design ability of middle school students' scientific creativity.

It is suggested that future researchers adopt multivariable design and follow-up study combined with experimental design, divide research samples into experimental group and control group, and pay attention to the development trend of creativity of middle school students in different school sports atmosphere and the difference of development level and speed between groups.

\section{Acknowledgments}

Key projects are funded by Xi'an Social Science Fund Planning Fund (Project approval number: 17T33). 


\section{References}

[1] Litwin G H, Stringer R A. Motivation and organizational climate. American Journal of Sociology. 1968; 82(4): 12201235.

[2] Peicun Cai. (1985) A comparative study of the relationship between principals' leadership traits, power base, school organizational structure and organizational climate and teachers' job satisfaction. National Chengchi University, Taipei.

[3] Weizheng Chen, Jinping Li. Review and Prospect of Tissue Climate Research. Foreign Economy and Management. 2005; 27(8): 18-25.

[4] Yuandong Gu, Jisheng Peng. The Impact of Organizational Innovation atmosphere on Employees' Innovative Behavior: The Mediating Role of Innovative Self-efficacy. Nankai Management Review. 2010; 13(1): 30-41.

[5] Weiping Hu, Guoliang Yu. Research on scientific creativity of middle school students. Education Research. 2002; (1): 44-48.

[6] Jinzhen Li, Wenzhong Wang, Jiannong Shi. The Development of Children's Practical Creativity and Its Relationship with Family Environment. Acta Psychologica Sinica. 2004; 36(6): 732-737.

[7] Anna H. Hung, Aaron M. Luebbe, Paul D., Flaspohler. Measuring School Climate: Factor Analysis and Relations to Emotional Problems, Conduct Problems, and Victimization in Middle School Students. School Mental Health. 2015; 7: 105-119.

[8] Nicholas A. G., Alvin L. The Meriden School Climate Survey-Student Version: Preliminary Evidence of Reliability and Validity. Assessment for Effective Intervention. 2016; 41(2): 67-78.

[9] Wang, M. T., \& Jessica L. Degol1. School Climate: a Review of the Construct, Measurement, and Impact on Student Outcomes. Educ Psychol Rev. 2016; 28: 315-352.

[10] Chongde Lin, Weiping Hu. Growth rules and training modes of creative talents. Journal of Beijing Normal University (social science edition). 2012; 1:36-41.

[11] Jiliang Shen, Weiping Hu, Chongde Lin. The Compilation of Juvenile Science Creativity Test. Psychological Development and Education. 2002; 4: 76-81.

[12] Amabile TM. Creativity in context. Boulder. Co: Westview Press; 1996.

[13] Bock G W, Zmud R W, Kim Y G, et al. Behavioral intention formation in knowledge sharing: examining the roles of extrinsic motivators, social-psychological factors, and organizational climate. Mis Quarterly. 2005; 29(1): 87-111.

[14] Yun Liu, Jintao Shi. Study on the Influence Process of Organizational Innovation Climate on Employees' Innovative Behavior - Based on the Analysis of the Mediating Effect of Psychological Authorization. China Soft Science. 2010; (3): 133-144.

[15] Xianya Wang, Sheng Lin, Liyun Chen et al. An Empirical Study on the Relationship between Organizational Atmosphere, Tacit Knowledge Sharing Behavior and Employees' Innovation Performance. Soft Science. 2014; 28(5): 4347.

[16] Minglong Wu. SPSS statistical application practice: questionnaire analysis and application statistics. Beijing: Science Press; 2003.

[17] William J. W. Creativity and high school climate. Gifted Child Quarterly. 1966; 10(3): 139-144.

[18] Chang, C. P., Hsu, C. T., Chen, I. J. The relationship between the playfulness climate in the classroom and student creativity. Qual Quant. 2013; 47: 1493-1510.

[19] Tseng, C.M. (2002) Graduate playfulness, humor, creative attitude, the perception created by the Department of the relationship between environment and creativity (Unpublished master thesis). National Taipei University, Taipei.

[20] Yanfang Li, Ying Wang. A Review of School Environmental Factors Affecting the Development of Children and Middle School Students' Creativity. China Special Education. 2009; 2: 80-84.

[21] Zhe Gong, Changshen Liu, Bing Wang, et al. Effects of trust on creativity: stimulation, inhibition and inverted U hypothesis. Progress in Psychological Science. 2017; 25(3): 463-474.

[22] Yunfeng Liu, Zhenyu Yao. The Influence of Tutors' Support Behavior on Graduate Students' Creativity: Taking Trust as a Disturbing Variable. Journal of Information. 2010; 29(6): 6-9.

[23] Fleith DDS. Teacher and student perceptions of creativity in the classroom environment. Roeper Review. 2000; 22(3): 148-153.

[24] Donaldson J., Hammrich P. Sports as a Creative Way to Teach Science. International Journal of Curriculum and Instruction. 2016; 8(2): 64-72. 